\title{
Impact of potential physiological changes due to COVID-19 home confinement on athlete health protection in elite sports: a call for awareness in sports programming
}

Sarto $\mathrm{F}^{1}$, Impellizzeri $\mathrm{FM}^{2}$, Spörri $\mathrm{J}^{3}$, Porcelli $\mathrm{S}^{4,5}$, Olmo $\mathrm{J}^{6}$, Requena $\mathrm{B}^{6}$, Suarez-Arrones $\mathrm{L}^{7}$, Arundale $\mathrm{A}^{8}$, Bilsborough $\mathrm{J}^{9}$, Buchheit $\mathrm{M}^{10}$, Clubb J ${ }^{11}$, Coutts $\mathrm{A}^{2}$, Nabhan $\mathrm{D}^{12}$, Torres Ronda $\mathrm{L}^{13}$, Mendez-Villanueva $\mathrm{A}^{14}$, Mujika ${ }^{15,16}$, Maffiuletti NA ${ }^{17}$, and Franchi MV ${ }^{1}$

${ }^{1}$ Institute of Physiology, Department of Biomedical Sciences, University of Padua, Italy

${ }^{2}$ Human Performance Research Centre, Faculty of Health, University of Technology Sydney, Australia

${ }^{3}$ Sports Medical Research Group, Department of Orthopaedics, Balgrist University Hospital, University of Zurich

${ }^{4}$ Department of Molecular Medicine, University of Pavia, Italy

${ }^{5}$ Sports Medicine Physician Team, Alpine Ski and Snowboard Team, Italian Winter Sports Federation

${ }^{6}$ Football Science Institute, Granada, Spain

${ }^{7}$ FC Basel 1983, Switzerland

${ }^{8}$ Brooklyn Nets, NBA, USA

${ }^{9}$ New England Patriots, NFL, USA

${ }^{10}$ Myorobie Performance, France

${ }^{11}$ Buffalo Bills, NFL, USA

${ }^{12}$ US Olympic and Paralympic committee, USA

${ }^{13}$ Philadelphia 76ers, NBA, USA

${ }^{14}$ Qatar Football Association, Qatar

${ }^{15}$ Department of Physiology, Faculty of Medicine and Nursing, University of the Basque Country, Leioa, Basque Country

${ }^{16}$ Exercise Science Laboratory, School of Kinesiology, Faculty of Medicine, Universidad Finis Terrae, Santiago, Chile

${ }^{17}$ Human Performance Lab, Schulthess Clinic, Zurich, Switzerland

This document is a pre-print.

Cite as:

Sarto, F., Impellizzeri, F. M., Spörri, J., Porcelli, S., Olmo, J., Requena, B., ... Franchi, M. V. (2020). Impact of potential physiological changes due to COVID-19 home confinement on athlete health protection in elite sports: a call for awareness in sports programming. Retrieved from osf.io/preprints/sportrxiv/4zb3a 
Correspondent Author:

Martino V. Franchi, PhD

Institute of Physiology, Department of Biomedical Sciences,

University of Padua, Italy

martino.franchi@unipd.it

ORCID: 0000-0003-3165-4536

Twitter:@FabioSarto3; @francoimpell; @MVFranchi

ARTICLE TYPE: Editorial

KEYWORDS: Elite Athletes; Injury Risk; Detraining; Skeletal Muscle; Injury Prevention

WORD COUNT: 754 
A global emergency called COVID-19 (coronavirus disease) has spread worldwide in early 2020. Preventive measures to reduce risk of infection include social distancing and the closing of commercial activities to avoid social gatherings. Elite sport is also tremendously affected: ongoing championships have been suspended and the major international events have been postponed (e.g. Summer Olympics, UEFA European Football Championship). This is the first time since the Second World War that all elite athletes are forced to interrupt competitions. Further, most elite athletes are forced to train at home, on their own and mostly unsupervised. Some elite sports clubs have provided players with home-based training programs and/or organized video conferences for online training sessions lead by their fitness trainers. However, logistical constraints, and the difficulty to implement sport-specific exercise strategies, make it difficult to provide training solutions comparable to those adopted under normal circumstances. During COVID-19 home confinement, athletes are likely exposed to some level of detraining (i.e. the partial or complete loss of training-induced morphological and physiological adaptations), as a consequence of insufficient and/or inappropriate training stimuli (1). Such changes may result in impaired performance and increased injury risk if, upon restart, an appropriate sport-specific reconditioning cannot be granted. Cardiorespiratory and neuromuscular adaptations are fundamental in different sports, and substantial declines are known to occur after short-term (<4 weeks) training cessation (1). Further, injury occurrence seems to be regulated by a complex mechanical interplay between tissue stress, strain, and loading (2). Therefore, alterations in mechanical structures, such as muscles and tendons, are likely involved in the injury process.

Lessons from physiological studies on muscle and tendon adaptations to unloading (such as bed rest and unilateral lower limb suspension, which can be considered as an extreme form of detraining) taught us that changes in muscle size and architecture, as well as in tendon mechanical properties, can occur in lower limb muscles even after exposure to short-term unloading (3). The rate of muscle disuse atrophy may be even more accelerated in elite athletes since highly trained subjects with greater initial muscle mass exhibit accentuated muscle loss(4). Besides morphological 
changes, reductions in muscle strength, power and rate of force development have been observed after short-term disuse $(3,5)$. Unfortunately, there is limited literature allowing a direct translation of such observations to elite sport, as the available evidence has focused on post-injury conditions (6), or on the effects of the off-season (7). Previous work can scarcely mimic the situation that athletes are experiencing now, with a sudden and longer than normal reduction in total training loads and the challenge to provide sport-specific stimuli. Nevertheless, we could rely on disuse-based studies to hypothesize that, in this period of activity reduction, muscles and tendons will undergo alterations of a similar nature. This will likely result in increased injury risks after COVID-19 home confinement release (2). There is a previous similar scenario after the National football League (NFL) lockout in 2011, where during a period over 3 months players underwent an uncommon offseason without normal access to their facilities and training resources, where a higher rate of Achiles tendon injuries occurred over the first perior of the training camp and the subsequent season (8).

To date, it is difficult to predict when elite sports will restart. Two different scenarios are possible. In the first one, the COVID-19 pandemic situation will improve relatively quickly and governance would permit to restart sports events behind closed doors. In this scenario, in order to conclude championships once the emergency will end, a lot of matches/events would be probably condensed in a short time and athletes may be unprepared to cope with the elevated training and match demands. For this reason, a sport-specific reconditioning period would be necessary for the athletes to recover their in-season neuromuscular and cardiorespiratory qualities, thus potentially reducing the risk of injury. In the second scenario, the emergency will continue, and championships will not be completed. This situation of insufficient and/or inadequate training would be protracted for several months and the associated physiological decline may be even more accentuated. In this case, a prolonged preseason would be warranted to allow full resurgence of athletes' physiological function and performance. 
With this commentary, we aim to recommend extreme caution in sports programming after the COVID-19 emergency and we advise to involve all stakeholders in the decisions (e.g. medical staff, head of performance, coaches, fitness trainers and players). We are still unsure when and how to restart championships and events, but we advise to consider the impact that choices could have on injury risk in elite athletes.

\section{Contributors}

FS, FMI and MVF designed and conceptualised the paper. FS, FMI, JS, SP, IM, NAM and MVF wrote the initial draft. LSA, AA, JB, MB, JC, AC, DN, LTR add AMV revised the draft and endorsed it in its final form. All authors approved the final version.

\section{Funding}

None received.

\section{Competing Interests}

None decalred.

\section{References}

1. Mujika I, Padilla S. Detraining: Loss of Training-Induced Physiological and Performance Adaptations. Part I: Short term insufficient training stimulus. Sport Med [Internet]. 2000;30(2):79-87. Available from:

https://link.springer.com/content/pdf/10.2165\%2F00007256-20003002000002.pdf\%0Ahttp://link.springer.com/10.2165/00007256-200030020-00002

2. Kalkhoven JT, Watsford ML, Impellizzeri FM. A conceptual model and detailed framework for stress-related, strain-related, and overuse athletic injury. J Sci Med Sport [Internet]. 2020; Available from: https://doi.org/10.1016/j.jsams.2020.02.002

3. de Boer MD, Maganaris CN, Seynnes OR, Rennie MJ, Narici M V. Time course of muscular, neural and tendinous adaptations to 23 day unilateral lower-limb suspension in 
young men. J Physiol. 2007;583(3):1079-91.

4. Miles MP, Heil DP, Larson KR, Conant SB, Schneider SM. Prior Resistance Training and Sex Influence Muscle Responses to Arm Suspension. Med Sci Sports Exerc. 1983;37(11):1983-9.

5. Rejc E, Floreani M, Taboga P, Botter A, Toniolo L, Cancellara L, et al. Loss of maximal explosive power of lower limbs after 2 weeks of disuse and incomplete recovery after retraining in older adults. J Physiol. 2018 Feb 15;596(4):647-65.

6. Milsom J, Barreira P, Brugess D, Iqbal Z, Morton J. Case Study: Muscle Atrophy and Hypertrophy in a Premier League Soccer Player During Rehabilitation From ACL Injury. Int J Sport Nutr Exerc Metab. 2014;24(5):543-52.

7. Suarez-Arrones L, Lara-Lopez P, Maldonado R, Torreno N, De Hoyo M, Nakamura FY, et al. The effects of detraining and retraining periods on fat-mass and fat-free mass in elite male soccer players. PeerJ. 2019;7.

8. Myer GD, Faigenbaum AD, Cherny CE, Heidt RS, Hewett TE. Did the NFL lockout expose the achilles heel of competitive sports? J Orthop Sports Phys Ther. 2011;41(10):702-5. 